\title{
A UNIVALENT FUNCTION NOWHERE SEMICONFORMAL ON THE UNIT CIRCLE
}

\author{
SHINJI YAMASHITA
}

\begin{abstract}
We shall construct a function $f$ holomorphic and univalent in the open unit disk such that $f$ is not semiconformal at any point of the unit circle. It is also shown that $f$ may be extended quasiconformally to the whole extended plane.
\end{abstract}

1. Introduction. Let $S$ be the family of all functions holomorphic and univalent in $D=\{|z|<1\}$. Then $f \in S$ is called conformal at a point $\zeta$ of $\Gamma=\{|z|=1\}$ if $f$ has the angular limit $f(\zeta) \neq \infty$ at $\zeta$ and if the function $\arg [(f(\zeta)-f(z)) /(\zeta-z)]$ of $z$ has a finite angular limit at $\zeta$ (see [5, p. 303]). Following D. M. Campbell and J. A. Pfaltzgraff [2, p. 74], we call $f \in S$ semiconformal at $\zeta \in \Gamma$ if the radial limit $f^{*}(\zeta)=\lim _{r \rightarrow 1-} f(r \zeta) \neq \infty$ exists, and if the function $\left(f^{*}(\zeta)-f(z)\right) /\left((\zeta-z) f^{\prime}(z)\right)$ of $z$ has the angular limit one at $\zeta$. It is known that if $f$ is conformal at $\zeta$, then $f$ is semiconformal at $\zeta$ [6], while the converse is false ([7, p. 85 with a few modifications]; see [4, p. 258] also). It is also known that there exists $f \in S$ such that $f$ is not conformal at any point of $\Gamma[5$, p. 304]. We shall prove the following theorem which extends the above italicized proposition.

THEOREM. There exists $f$ holomorphic and univalent in $D$ such that $f$ is not semiconformal at any point of $\Gamma$.

\section{Proof of Theorem.}

LEMMA 1. A function $f \in S$ is semiconformal at $\zeta \in \Gamma$ if and only if

$$
\lim (\zeta-z) f^{\prime \prime}(z) / f^{\prime}(z)=0
$$

as $z \rightarrow \zeta$ in each Stolz angle at $\zeta$.

To prove Lemma 1 it suffices to consider the case $\zeta=1$. We note that $f$ is semiconformal at 1 if and only if the same is true of $F$ defined by

$$
F(z)=(f(z)-f(0)) / f^{\prime}(0), \quad z \in D .
$$

According to C. Pommerenke [4, Theorem 3.15, p. 257] $F$ is semiconformal at 1 if and only if

$$
F(z, \xi) \rightarrow z /(1+z)
$$

as $0<\xi \rightarrow 1-$ locally uniformly in $D$, where

Received by the editors July 5, 1977.

AMS (MOS) subject classifications (1970). Primary 30A36, 30A60.

๑) American Mathematical Society 1978 


$$
F(z, \xi)=\frac{F((z+\xi) /(1+\xi z))-F(\xi)}{\left(1-\xi^{2}\right) F^{\prime}(\xi)}
$$

in effect, Pommerenke [4, p. 257] adopted (2.2) as the definition of semiconformality at 1 . Lemma 1 now follows from the first half of [4, Theorem 3.14, p. 255], combined with the identity $F^{\prime \prime} / F^{\prime}=f^{\prime \prime} / f^{\prime}$.

LEMMA 2 (P. LAPPAN [3, p. 113]). There exists a function $g$ holomorphic in $D$ which satisfies the following:

$$
\begin{gathered}
\sup _{z \in D}(1-|z|)|g(z)| \leqslant 2 ; \\
\underset{r \rightarrow 1-}{\lim \sup }(1-r)|g(r \zeta)|>0
\end{gathered}
$$

at each $\zeta \in \Gamma$.

For the proof of our theorem we set

$$
f(z)=\int_{0}^{z} \exp \left(\int_{0}^{w} g(\eta) / 5 d \eta\right) d w
$$

so that

$$
f^{\prime \prime} / f^{\prime}=g / 5 \text {. }
$$

Then it follows from (2.3) and (2.5) that

$$
\sup _{z \in D}\left(1-|z|^{2}\right)\left|f^{\prime \prime}(z) / f^{\prime}(z)\right| \leqslant 4 / 5<1 \text {. }
$$

Therefore $f$ is univalent in $D$ by the result of J. Becker [1, Corollary 4.1, p. 36]. Furthermore, (2.1) is false at each $\zeta \in \Gamma$ because of (2.4) and (2.5). We now conclude that $f$ is not semiconformal at any point of $\Gamma$.

REMARK. Actually, $f$ with (2.6) admits a quasiconformal extension to the whole extended plane by the cited result of Becker [1, Corollary 4.1, p. 36]. Therefore quasiconformality does not imply semiconformality.

\section{REFERENCES}

1. J. Becker, Löwnersche Differentialgleichung und quaikonform fortsetzbare schlichte Funktionen, J. Reine Angew. Math. 255 (1972), 23-43.

2. D. M. Campbell and J. A. Pfaltzgraff, Boundary behaviour and linear invariant families, J. Analyse Math. 29 (1976), 67-92.

3. P. Lappan, Fatou points of harmonic normal functions and uniformly normal functions, Math. Z. 102 (1967), 110-114.

4. C. Pommerenke, Linear-invariante Familien analytischer Funktionen. II, Math. Ann. 156 (1964), 226-262.

5. Univalent functions, Studia Mathematica/Mathematische Lehrbücher, vol. 25, Vandenhoeck und Ruprecht, Göttingen, 1975.

6. C. Visser, Uber beschränkte analytische Funktionen und die Randverhältnisse bei konformen Abbildungen, Math. Ann. 107 (1933), 28-39.

7. J. L. Walsh and D. Gaier, Zur Methode der variablen Gebiete bei der Randverzerrung, Arch. Math. 6 (1955), 77-86.

Department of Mathematics, Tokyo Metropolitan University, Fukazawa 2-1-1, SeTAGAYA-KU, TOKYO 158, JAPAN 